\title{
Thermal conductance as a probe of the nonlocal order parameter for a topological superconductor with gauge fluctuations
}

\author{
B. van Heck, ${ }^{1}$ E. Cobanera, ${ }^{1}$ J. Ulrich, ${ }^{2}$ and F. Hassler ${ }^{2}$ \\ ${ }^{1}$ Instituut-Lorentz, Universiteit Leiden, P.O. Box 9506, 2300 RA Leiden, The Netherlands \\ ${ }^{2}$ Institute for Quantum Information, RWTH Aachen University, 52056 Aachen, Germany \\ (Received 15 January 2014; revised manuscript received 4 April 2014; published 21 April 2014)
}

\begin{abstract}
We investigate the effect of quantum phase slips on a helical quantum wire coupled to a superconductor by proximity. The effective low-energy description of the wire is that of a Majorana chain minimally coupled to a dynamical $\mathbb{Z}_{2}$ gauge field. Hence the wire emulates a matter-coupled gauge theory, with fermion parity playing the role of the gauged global symmetry. Quantum phase slips lift the ground-state degeneracy associated with unpaired Majorana edge modes at the ends of the chain, a change that can be understood as a transition between the confined and the Higgs-mechanism regimes of the gauge theory. We identify the quantization of thermal conductance at the transition as a robust experimental feature separating the two regimes. We explain this result by establishing a relation between thermal conductance and the Fredenhagen-Marcu string order parameter for confinement in gauge theories. Our work indicates that thermal transport could serve as a measure of nonlocal order parameters for emergent or simulated topological quantum order.
\end{abstract}

DOI: 10.1103/PhysRevB.89.165416

PACS number(s): 74.25.fc, 11.15.Ha, 74.78.-w, 75.10.Pq

Topological phases of matter cannot be characterized by any local order parameter and, hence, signatures of these phases are not accessible by a local experimental probe. For free fermions, the complete classification of topological phases has recently been established [1-3] and a connection between the (experimentally accessible) linear response properties of a system and the value of its topological invariant has been obtained. A prominent and illustrative example are one-dimensional (1D) topological superconductors [4-7], currently the subject of intense theoretical $[8,9]$ and experimental investigation [10-15]. In this case, the topological phase is characterized by unpaired Majorana zero modes at the ends of the superconductor, whose presence allows nonlocal storage of one bit of quantum information encoded in the total fermion parity of the superconductor [4]. This topological phase can be recognized by striking transport properties [9]. Perfect Andreev reflection off a Majorana end mode leads to a quantized zero-bias conductance of $G_{0}=2 e^{2} / h$ [16-19]. The peak can only be removed if the system undergoes a phase transition into a phase without Majorana modes. Exactly at the transition, the two unpaired Majorana modes combine into a perfectly transmitting mode. As a consequence, the thermal conductance through the wire peaks at a value equal to its superconducting quantum $K_{0}=\pi^{2} k_{B}^{2} T / 6 h$ at temperature $T$ [20]. The quantization of the peak is a way to identify the topological phase transition, even in a wire of finite size [20]. In the topologically trivial phase, both zero-bias Andreev and thermal conductance are zero.

It is currently a challenge in condensed matter physics to extend the classification of topological phases to interacting fermionic systems (see Refs. [21-23]) and in particular, to provide a similar connection with experimental probes. Often, insight into interacting topological phases is offered by nonlocal order parameters [24,25]. However, such quantities lack an obvious thermodynamic meaning and do not enable natural mean-field approximations. If available, they are useful theoretical tools [26,27], without direct experimental implications. Thus, not surprisingly, they are dubbed "hidden."

In this paper, we will show that nonlocal order parameters can be directly linked to transport properties in the linear response regime. We will show this for the case of a 1D topological superconductor subject to quantum phase slips (see Fig. 1). The system is described by an effective interacting Hamiltonian akin to a matter-coupled lattice gauge theory, the 1D $\mathbb{Z}_{2}$ Higgs model [28]. A nonlocal order parameter is in this case known: the Fredenhagen-Marcu string order parameter [29], originally proposed as a criterion for confinement [30] and recently revisited in the context of topological order [31]. We will show that the Fredenhagen-Marcu order parameter is connected in our system to a simple transport coefficient, the thermal conductance.

Let us start by discussing the role of quantum phase slips (QPS) in topological superconductors. QPS are quantum tunneling events where the phase of the superconducting order parameter changes locally by $2 \pi$. In $1 D$, QPS destroy the superconducting phase at zero temperature [32-34] and thus remove the topological protection of a Majorana qubit [35], since the latter presupposes the superconducting ordering which breaks the electromagnetic U(1) symmetry down to $\mathbb{Z}_{2}$. For $d$-dimensional superconductors with $d>1$, QPS are suppressed as they generate a domain wall in the superconducting order parameter, leading to $\kappa \propto \exp \left[-(L / \xi)^{d-1}\right]$, with $L$ the linear dimension of the system and $\xi$ the coherence length. In this sense, Kitaev's model of topological protection is not purely one-dimensional, since a bulk (three-dimensional) superconductor is crucial for achieving the fault tolerance of a Majorana qubit [35].

To study QPS in a concrete setting, we follow the approach of Ref. [36] and consider a chain of coupled superconducting islands, with superconducting phase $\phi_{m}$, placed on top of a nanowire or of a quantum spin Hall edge (see Fig. 1). The junctions between the islands then naturally form weak links through which QPS happen. The Euclidean action describing 


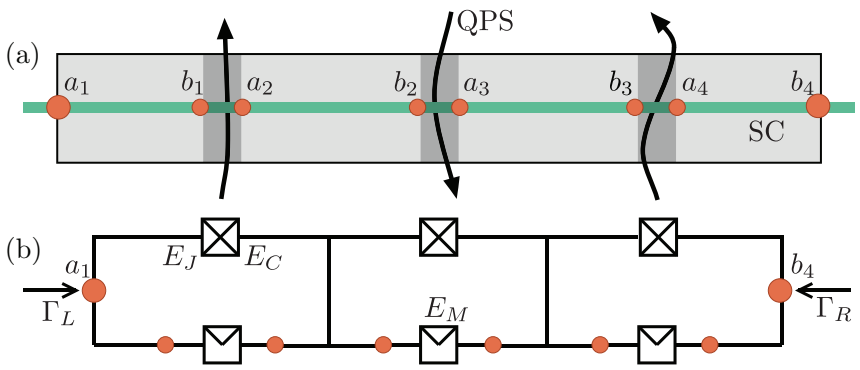

FIG. 1. (Color online) Panel (a): An $s$-wave superconductor (gray) is deposited on top of a helical quantum wire (green), which can be, for example, a semiconducting nanowire or the edge of a quantum spin Hall insulator. We consider the effect of quantum phase slips in the superconductor (black arrows). Once a moderate magnetic field is applied to break time reversal invariance, Majorana modes (orange dots) appear at the ends of the wire and at weak links when the phase slips happen. Panel (b): We show an equivalent circuit describing the system [see Eq. (1)]. Here, as usual, a box with a cross denotes a Josephson junction and its capacitance. On the other hand, a box with only half of a cross denotes the $4 \pi$-periodic Majorana junction. Arrows represent coupling to external leads to the Majorana modes at the end with strength $\Gamma_{L}$ and $\Gamma_{R}$.

a chain of $N$ islands reads $\mathcal{S}=\int_{0}^{1 / T} \mathcal{L} d t$, with [37,38]

$$
\begin{aligned}
\mathcal{L}= & \sum_{m=1}^{N-1}\left[\frac{\dot{\varphi}_{m}^{2}}{2 E_{C}}+E_{J}\left(1-\cos \varphi_{m}\right)\right. \\
& \left.-i E_{M} b_{m} a_{m+1} \cos \left(\varphi_{m} / 2\right)\right]-\sum_{m=1}^{N} i h a_{m} b_{m} ;
\end{aligned}
$$

here, $\varphi_{m}=\phi_{m+1}-\phi_{m}$ is the phase difference across each junction. The charging energy $E_{C}=e^{2} / 2 C$ and the Josephson energy $E_{J}=\hbar I_{c} / 2 e$ are respectively determined by the capacitance $C$ and the critical current $I_{c}$ of the junction. A topological superconducting wire hosts two Majorana zeroenergy modes $a_{m}, b_{m}$ on each island. They are responsible for the term proportional to $E_{M}$ in (1), describing tunneling of individual electrons [5]. The Hermitian operators $a_{m}, b_{m}$ obey the anticommutation rules $\left\{a_{m}, b_{n}\right\}=0$ and $\left\{a_{m}, a_{n}\right\}=\left\{b_{m}, b_{n}\right\}=2 \delta_{m n}$. Additionally, the finite size of the islands leads to an overlap between Majorana modes and an associated energy splitting denoted by $h$. The local fermion parity $p_{m}= \pm 1$ at each junction is defined via the occupation number of a fermionic mode $c_{m}=\frac{1}{2}\left(b_{m}-i a_{m+1}\right)$ as $p_{m}=1-2 c_{m}^{\dagger} c_{m}=i b_{m} a_{m+1}$. The total fermion parity operator $(-1)^{F}=i a_{1} \prod_{m=1}^{N-1} p_{m} b_{N}$ is a global symmetry of the system.

Different from previous studies [39-41], we are interested in the regime $E_{J} \gg E_{M}, E_{C}, h$, where the superconducting phase difference at any junction can only be a multiple of $2 \pi$, due to the large Josephson energy. The relevant quantum fluctuations in the chain are QPS connecting classical minima, whose amplitude $\kappa \simeq\left(E_{C} E_{J}^{3}\right)^{1 / 4} \exp \left(-8 \sqrt{E_{J} / E_{C}}\right)$ can be computed in the semiclassical approximation [36]. A shift of $\varphi_{m}$ by $2 \pi$ changes the sign of $E_{M} \cos \left(\varphi_{m} / 2\right)$ and thus it also changes the energetically favored value of the junction parity $p_{m}$ [37]. In this regime, the value of $\cos \left(\varphi_{m} / 2\right)$ is reduced to a
$\mathbb{Z}_{2}$ quantum degree of freedom. The effective Hamiltonian of the chain,

$$
H=-\sum_{m=1}^{N} i h a_{m} b_{m}-\sum_{m=1}^{N-1}\left[i E_{M} b_{m} a_{m+1} \tau_{m}^{z}+\kappa \tau_{m}^{x}\right],
$$

describes Majorana modes coupled to $N-1$ Pauli matrices $\tau_{m}^{z}=\cos \left(\varphi_{m} / 2\right)$, one per junction [26]. The last term in the Hamiltonian describes QPS that change $\varphi_{m}$ by $2 \pi$ at a rate $\kappa / \hbar$.

In the absence of fluctuations of the superconducting order parameter, that is, at $\kappa=0$, we recover the Kitaev model. In this case, the $\tau_{m}^{z}$ degrees of freedom are redundant. The Hamiltonian $H$ can be block diagonalized by freezing them in some classical configuration. All blocks in this decomposition have identical energy spectra. For any classical configuration of the spins $\tau_{m}^{z}$, a quantum critical point at $h=E_{M}$ separates a topologically nontrivial phase at $h<E_{M}$ from the trivial phase at $h>E_{M}$. The nontrivial phase has a twofold ground-state degeneracy if both even and odd total fermion parities $(-1)^{F}= \pm 1$ are considered, signaling the presence of unpaired Majorana modes at either ends of the chain.

The interaction of the fluctuating superconducting phase with the Majorana modes is such that, for each island, a local symmetry $C_{m}$ of $H$ emerges, given by

$$
\begin{aligned}
& C_{1}=i a_{1} b_{1} \tau_{1}^{x}, \quad C_{N}=\tau_{N-1}^{x} i a_{N} b_{N}, \\
& C_{m}=\tau_{m-1}^{x} i a_{m} b_{m} \tau_{m}^{x} \quad(m=2, \ldots, N-1) .
\end{aligned}
$$

These local symmetries are gauge symmetries and appear because the phase difference and fermion parity of a junction are not independent degrees of freedom [42]: a change in the occupation number of the fermionic mode $c_{m}$ is equivalent to advancing the phase $\varphi_{m}$ by $2 \pi$. As a result, the global fermion parity $(-1)^{F}$ can be expressed as a product of the local gauge symmetries $(-1)^{F}=\prod_{m=1}^{N} C_{m}$ [26]. It follows that the $\tau_{m}^{z}$ play the role of a $\mathbb{Z}_{2}$ gauge field, minimally coupled to the fermionic degrees of freedom and with dynamics generated by the QPS.

The link to lattice field theories can be made more explicit. Our effective Hamiltonian $H$ of Eq. (2) can be interpreted as an approximation to the lattice-regularized 1D Higgs model [28] given by

$$
H_{\mathrm{H}}=-\sum_{m=1}^{N} \frac{\lambda}{2} \partial_{\phi_{m}}^{2}+\sum_{m=1}^{N-1}\left[-\frac{g^{2}}{2} \partial_{\theta_{m}}^{2}+v^{2} \cos \left(\varphi_{m}-\theta_{m}\right)\right] .
$$

This Hamiltonian follows by standard techniques $[43,44]$ from the Euclidean action of the Higgs model of Ref. [28]. Here, the angular variables $\phi_{m}, \theta_{m}$ represent the Higgs and electromagnetic gauge field, respectively. The parameter $v^{2}$ is the vacuum expectation value of the Higgs field in the brokensymmetry state. The parameters $\lambda, g^{2}$ control the strength of the fluctuations of the matter and gauge fields.

Our Hamiltonian $H$ is obtained from that of the Higgs model $H_{\mathrm{H}}$ by using the approximation $-\pi^{2} \partial_{x}^{2} / 2 \approx$ $\cos \left(\pi \partial_{x}\right)-1$ for $x=\phi_{m}, \theta_{m}$ and truncating the angular variables to the values $\phi_{m}, \theta_{m} \in\{0, \pi\}$. Within the truncated Hilbert space, $\cos \left(\pi \partial_{\phi_{m}}\right)=\sigma_{m}^{x}$ and $\cos \left(\pi \partial_{\theta_{m}}\right)=\tau_{m}^{x}$. Hence, $H_{\mathrm{H}}$ reduces (up to an irrelevant additive constant) to the spin 
chain Hamiltonian

$$
H_{\mathbb{Z}_{2}}=\sum_{m=1}^{N} \frac{\lambda}{\pi^{2}} \sigma_{m}^{x}+\sum_{m=1}^{N-1}\left[\frac{g^{2}}{\pi^{2}} \tau_{m}^{x}+v^{2} \sigma_{m}^{z} \tau_{m}^{z} \sigma_{m+1}^{z}\right] .
$$

The Hamiltonian $H_{\mathbb{Z}_{2}}$ is precisely that of the $\mathbb{Z}_{2}$ Higgs model [28]. Finally, the Jordan-Wigner transformation $a_{m}=$ $\sigma_{m}^{x} \prod_{j=1}^{m-1} \sigma_{j}^{z}, b_{m}=\sigma_{m}^{y} \prod_{j=1}^{m-1} \sigma_{j}^{z}$ shows that the $\mathbb{Z}_{2}$ Higgs model is equivalent to our Hamiltonian $H$, provided we identify $h=-\lambda / \pi^{2}, \kappa=-g^{2} / \pi^{2}$, and $E_{M}=-v^{2}$ [45].

As our effective Hamiltonian $H$ is related to the Higgs model, we might expect the Higgs mechanism to be present. As a result, gapless excitations should become gapped for arbitrarily small values of $\kappa$, that is, for arbitrarily weak fluctuations of the superconducting order parameter. In other words, the small but finite charging energy $E_{C}$ of each island breaks the ground-state degeneracy and splits the otherwise unpaired Majorana modes. In this way, the Higgs mechanism offers a way to locally break the topological degeneracy of the Majorana chain. It is known that this expectation is indeed correct in the thermodynamic limit, as at $\kappa \neq 0$ the Hamiltonian (4) has no phase transitions and describes a gapped phase with a single ground state $[26,28,46]$. However, in a finite chain signatures of the topologically nontrivial phase, which is present at $\kappa=0$ and $h<E_{M}$, should survive up to a finite value of $\kappa$. If this is true, then the Hamiltonian of a finite chain should be gapless along a line in the $(h, \kappa)$ plane.

In the following, we will show that in the linear response regime, the topological transition reflects itself in the thermal conductance $K$ through the system also at finite $\kappa$, whereas upon increasing $\kappa$, the local probe of Andreev conductance $G$ quickly becomes blind to it. To this end, we couple the left and right end of the chain with Hamiltonian $H$ to normal leads through tunneling Hamiltonians [42] $H_{\mathrm{L}}=\gamma_{\mathrm{L}} c_{\mathrm{L}}^{\dagger} a_{1} e^{-i \phi_{1} / 2}+$ H.c., $H_{\mathrm{R}}=\gamma_{\mathrm{R}} c_{\mathrm{R}}^{\dagger} b_{N} e^{-i \phi_{N} / 2}+$ H.c.; here, $\gamma_{\mathrm{L}}$ and $\gamma_{\mathrm{R}}$ denote the amplitudes for tunneling events into the left (L) and right $(\mathrm{R})$ leads, and $c_{\mathrm{L}}^{\dagger}, c_{\mathrm{R}}^{\dagger}$ are the creation operators for electrons in the noninteracting leads. We fix the gauge by choosing $\phi_{1}=0$, so that $\phi_{N}=\sum_{m=1}^{N-1} \varphi_{m}$. In the low-energy limit, $e^{-i \phi_{N} / 2}=$ $\prod_{m=1}^{N-1} \tau_{m}^{z}$, so we get

$$
H_{\mathrm{L}}=\gamma_{\mathrm{L}}\left(c_{\mathrm{L}}^{\dagger}+c_{\mathrm{L}}\right) a_{1}, \quad H_{\mathrm{R}}=\gamma_{\mathrm{R}}\left(c_{\mathrm{R}}^{\dagger}+c_{\mathrm{R}}\right) b_{N} \prod_{m=1}^{N-1} \tau_{m}^{z} .
$$

The tunneling Hamiltonians must break one of the gauge symmetries, since a tunneling event changes the total fermion parity. Due to our gauge choice, we obtain $\left\{H_{\mathrm{L}, \mathrm{R}}, C_{1}\right\}=0$, while $\left[H_{\mathrm{L}, \mathrm{R}}, C_{m}\right]=0$ for $m=2, \ldots, N$.

The Andreev conductance $G$ is determined by the charge transport across a normal metal-superconductor interface. To compute $G$, we set $\gamma_{\mathrm{R}}=0, \gamma_{\mathrm{L}}=\gamma$, apply a bias voltage $V$ to the left lead, and ground the rightmost superconducting island. In contrast, the thermal conductance $K$ is determined by the heat transport between two normal leads. To compute $K$ we set $\gamma_{\mathrm{L}}=\gamma_{\mathrm{R}}=\gamma$ and establish a small temperature difference between the right lead at temperature $T$ and the left lead at temperature $T+\delta T$. In the limit $T, V \rightarrow$ 0 , we obtain $G$ as $G=G_{0} \Gamma \operatorname{Im}\left[G_{11}(0)\right]$ [47] and $K$ as $K=4 K_{0} \Gamma^{2}\left|G_{1 N}(0)\right|^{2}[48]$, in terms of the tunnel coupling
$\Gamma=2 \pi|\gamma|^{2} \rho_{0}$ to a wide-band lead with density of states $\rho_{0}$ and the retarded Green's functions,

$$
\begin{aligned}
& G_{11}(\omega)=-i \int_{0}^{\infty} d t e^{i \omega t}\left\langle\left\{a_{1}(t), a_{1}(0)\right\}\right\rangle, \\
& G_{1 N}(\omega)=-i \int_{0}^{\infty} d t e^{i \omega t}\left\langle\left\{b_{N}(t) \prod_{m=1}^{N-1} \tau_{m}^{z}(t), a_{1}(0)\right\}\right\rangle .
\end{aligned}
$$

The averages in Eqs. (6) are taken over the ground-state wave function $|0\rangle$ of our effective Hamiltonian $H$. For any $\kappa, h, E_{M}>0$, the ground state of $H$ is unique and belongs to the gauge-invariant sector with $C_{m}|0\rangle=|0\rangle$ for all $m$ [49]. The time evolution in Eqs. (6) is determined by the total Hamiltonian $H_{\text {tot }}=H+H_{\mathrm{R}}+H_{\mathrm{L}}$. The retarded Green's function $G_{11}(t)=\int(d \omega / 2 \pi) e^{-i \omega t} G_{11}(\omega)$ is the amplitude for a reflection process whereby an electron enters the chain from the left lead at time $t_{i}=0$ and exits again from the left lead after a time $t_{f}=t$. Similarly, $G_{1 N}(t)$ is the amplitude for a transmission process whereby the electron enters at $t_{i}=0$ from the left lead and exits from the right lead after a time $t_{f}=t$.

We highlight that the thermal transport probes nonlocal quasiparticle transfer processes through the chain, characterized by the string correlator $G_{1 N}(t)=$ $-i\left\langle\left\{b_{N}(t) \prod_{m=1}^{N-1} \tau_{m}^{z}(t), a_{1}(0)\right\}\right\rangle$, which is a generalization of the conventional correlator $-i\left\langle\left\{b_{N}(t), a_{1}(0)\right\}\right\rangle$ studied in the context of the Majorana chain without the gauge degrees of freedom [17]. Due to the presence of the gauge string $\prod_{m=1}^{N-1} \tau_{m}^{z}$, the Green's function $G_{1 N}$ is similar to the Fredenhagen-Marcu string-order parameter [29],

$$
M_{\mathrm{FM}}=-i\left\langle b_{N} \prod_{m=1}^{N-1} \tau_{m}^{z} a_{1}\right\rangle
$$

which measures the presence of the topological phase in the model with fluctuation gauge degrees of freedom. In the following we probe this relation numerically.

To calculate the Green's function $G_{m n}(\omega)$, we follow the approach [50] of decoupling the gauged Majorana chain from the leads to first order in the lead coupling $\Gamma$ and neglecting higher-order (co-)tunneling processes. The bare Green's functions without the leads are calculated by exact diagonalization of the Hamiltonian (2) using a Lehmann spectral representation in terms of the exact eigenstates. The presence of the symmetries (3) greatly simplifies the task of computing $G_{m n}$. In fact, we only need to know the energy and the wave function of the ground state $|0\rangle$ and of all the states $|\psi\rangle$ such that $C_{1}|\psi\rangle=-|\psi\rangle$ while $C_{m \neq 1}|\psi\rangle=$ $|\psi\rangle$. Indeed, since $C_{1}$ is the only symmetry of $H$ which does not commute with the tunneling Hamiltonian $H_{\mathrm{L}, \mathrm{R}}$ but anticommutes instead, these are the only excited states to which transitions from the ground states are possible upon tunneling of an electron from the leads. For a chain of $N$ islands, there are $2^{N-1}$ of these states-against a total Hilbert space dimension of $2^{2 N-1}$ [51].

The numerical results for a chain of $N=11$ islands are shown in Fig. 2. At $\kappa=0$, coherently with known results, we observe an Andreev reflection plateau at $G_{0}$ in the nontrivial regime and a thermal conductance peak at the transition, which appears shifted to $h \simeq E_{M}\left(\Gamma / E_{M}\right)^{1 / N}$ due to finite chain size 

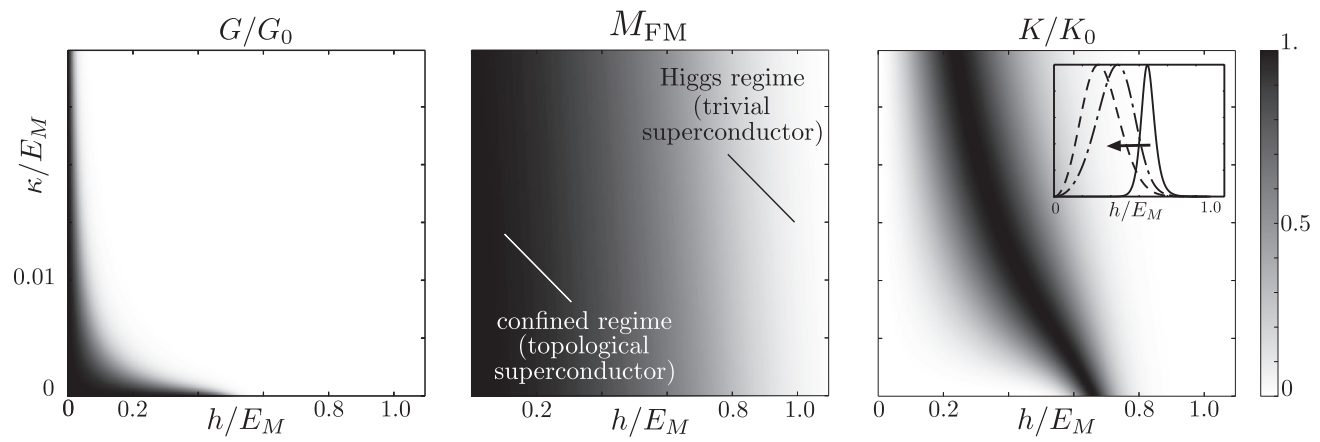

FIG. 2. Numerical results: Andreev conductance (left, in units of $G_{0}=2 e^{2} / h$ ), Fredenhagen-Marcu order parameter $M_{\mathrm{MF}}$ (center), and thermal conductance (right, in units of $K_{0}=\pi^{2} k_{B}^{2} T / 6 h$ ) plotted as a function of $h$ and $\kappa$. The results are obtained for a chain of $N=11$ islands with coupling constant $\Gamma=0.01 E_{M}$ to the leads, in the limit of vanishing temperature $T$ and small applied voltage $V$. The Andreev conductance $G$ is averaged over a small voltage interval $10^{-4} E_{M}$ to account for the finite-size energy splitting of the Majorana modes, as for a finite-size wire we trivially have $G=0[17,41]$. The Andreev conductance only shows a signal along the axis. On the other hand, the string-order parameter allows us to distinguish a confined regime - corresponding to the topological regime with Majorana end modes-from the trivial Higgs regime. The separation between these two regimes can be clearly identified by the peak in the thermal conductance. The inset in the right panel shows line cuts of the thermal conductance at $\kappa / E_{M}=0,0.01,0.02$, going from right to left as shown by the arrow. Due to finite-size effects, the transition at $\kappa=0$ is shifted from $h / E_{M}=1$ to $h / E_{M} \simeq\left(\Gamma / E_{M}\right)^{1 / 11} \simeq 0.7$, as expected. Increasing $\kappa$, the topological regime shrinks and only the Higgs regime survives.

and coupling to the leads. At finite $\kappa$, the Andreev plateau is quickly suppressed, except close to $h=0$, a limit where two isolated Majorana modes are always present. However, the quantized peak in thermal conductance persists in the interacting part of the parameter space, indicating the presence of a gapless transmitting mode and hence a strong signature of the existence of a topological regime. In fact, the position of the thermal conductance peak qualitatively follows the line of maximum change in the order parameter. We have checked that the agreement persists when varying the system size $N$.

To conclude, we have shown that QPS in a Majorana chain implement the $\mathbb{Z}_{2}$ Higgs model, where the fluctuations of the gauge field are determined by the rate $\kappa / \hbar$ for QPS. QPS locally destroy the topological phase of the Kitaev model at fixed fermion parity via a $\mathbb{Z}_{2}$ version of the Higgs mechanism. However, for finite system size and small $\kappa$, signatures of the topological phase remain visible in the thermal conductance through the system. The reason is that it is linked to the Fredenhagen-Marcu order parameter for the $\mathbb{Z}_{2}$ Higgs theory, which indicates the topological regime with gauge fluctuations present. The thermal conductance provides a clear transport signature of the transition from the topological to the trivial regimes in the presence of the interactions with the gauge field, whereas no signature of the transition is present in the Andreev conductance at a finite rate of QPS. Our results suggest that in topological quantum matter, bulk transport measurements offer access to nonlocal order parameters, just like susceptibility measurements do for local order parameters in broken-symmetry phases. It remains an interesting question for further studies how this scenario can be generalized to higher dimensions and non-Abelian gauge fields.

We thank Anton Akhmerov, Carlo Beenakker, Gerardo Ortiz, and Dirk Schuricht for fruitful discussions. B.vH. and E.C. were supported by the Dutch Foundation for Fundamental Research on Matter (FOM), the Netherlands Organization for Scientific Research (NWO/OCW), and an ERC Synergy Grant. J.U. and F.H. are grateful for support from the Alexander von Humboldt foundation and from the RWTH Aachen Seed Funds.
[1] A. Y. Kitaev, AIP Conf. Proc. 1134, 22 (2009).

[2] S. Ryu, A. P. Schnyder, A. Furusaki, and A. W. W. Ludwig, New J. Phys. 12, 065010 (2010).

[3] X. G. Wen, Phys. Rev. B 85, 085103 (2012).

[4] A. Y. Kitaev, Phys.-Usp. 44, 131 (2001).

[5] L. Fu and C. L. Kane, Phys. Rev. B 79, 161408 (2009).

[6] R. M. Lutchyn, J. D. Sau, and S. Das Sarma, Phys. Rev. Lett. 105, 077001 (2010).

[7] Y. Oreg, G. Refael, and F. von Oppen, Phys. Rev. Lett. 105, 177002 (2010).

[8] J. Alicea, Rep. Prog. Phys. 75, 076501 (2012).

[9] C. Beenakker, Annu. Rev. Condens. Matter Phys. 4, 113 (2013).

[10] V. Mourik, K. Zuo, S. M. Frolov, S. R. Plissard, E. P. A. M. Bakkers, and L. P. Kouwenhoven, Science 336, 1003 (2012).
[11] A. Das, Y. Ronen, Y. Most, Y. Oreg, M. Heiblum, and H. Shtrikman, Nat. Phys. 8, 887 (2012).

[12] M. T. Deng, C. L. Yu, G. Y. Huang, M. Larsson, P. Caroff, and H. Q. Xu, Nano Lett. 12, 6414 (2012).

[13] L. P. Rokhinson, X. Liu, and J. K. Furdyna, Nat. Phys. 8, 795 (2012).

[14] I. Knez, R.-R. Du, and G. Sullivan, Phys. Rev. Lett. 109, 186603 (2012).

[15] S. Hart, H. Ren, T. Wagner, P. Leubner, M. Mühlbauer, C. Brüne, H. Buhmann, L. W. Molenkamp, and A. Yacoby, arXiv:1312.2559.

[16] K. T. Law, P. A. Lee, and T. K. Ng, Phys. Rev. Lett. 103, 237001 (2009).

[17] K. Flensberg, Phys. Rev. B 82, 180516 (2010). 
[18] M. Wimmer, A. R. Akhmerov, J. P. Dahlhaus, and C. W. J. Beenakker, New J. Phys. 13, 053016 (2011).

[19] L. Fidkowski, J. Alicea, N. H. Lindner, R. M. Lutchyn, and M. P. A. Fisher, Phys. Rev. B 85, 245121 (2012).

[20] A. R. Akhmerov, J. P. Dahlhaus, F. Hassler, M. Wimmer, and C. W. J. Beenakker, Phys. Rev. Lett. 106, 057001 (2011).

[21] L. Fidkowski and A. Y. Kitaev, Phys. Rev. B 81, 134509 (2010).

[22] X.-G. Wen, Phys. Rev. B 89, 035147 (2014).

[23] L.-Y. Hung and X.-G. Wen, Phys. Rev. B 89, 075121 (2014).

[24] F. J. Wegner, J. Math. Phys. 12, 2259 (1971).

[25] Z. Nussinov and G. Ortiz, Ann. Phys. 324, 977 (2009).

[26] E. Cobanera, G. Ortiz, and Z. Nussinov, Phys. Rev. B 87, 041105 (2013).

[27] Y. Bahri and A. Viswanath, arXiv:1303.2600.

[28] E. Fradkin and S. H. Shenker, Phys. Rev. D 19, 3682 (1979).

[29] K. Fredenhagen and M. Marcu, Commun. Math. Phys. 92, 81 (1983).

[30] K. Fredenhagen and M. Marcu, Phys. Rev. Lett. 56, 223 (1986).

[31] K. Gregor, D. A. Huse, R. Moessner, and S. L. Sondhi, New J. Phys. 13, 025009 (2011).

[32] A. D. Zaikin, D. S. Golubev, A. van Otterlo, and G. T. Zimányi, Phys. Rev. Lett. 78, 1552 (1997).

[33] C. N. Lau, N. Markovic, M. Bockrath, A. Bezryadin, and M. Tinkham, Phys. Rev. Lett. 87, 217003 (2001).

[34] D. S. Golubev and A. D. Zaikin, Phys. Rev. B 64, 014504 (2001).

[35] J. D. Sau, B. I. Halperin, K. Flensberg, and S. Das Sarma, Phys. Rev. B 84, 144509 (2011).

[36] K. A. Matveev, A. I. Larkin, and L. I. Glazman, Phys. Rev. Lett. 89, 096802 (2002).
[37] D. Pekker, C.-Y. Hou, D. L. Bergman, S. Goldberg, İ. Adagideli, and F. Hassler, Phys. Rev. B 87, 064506 (2013).

[38] D. Pekker, C.-Y. Hou, V. E. Manucharyan, and E. Demler, Phys. Rev. Lett. 111, 107007 (2013).

[39] V. Shivamoggi, G. Refael, and J. E. Moore, Phys. Rev. B 82, 041405 (2010).

[40] C. Xu and L. Fu, Phys. Rev. B 81, 134435 (2010).

[41] F. Hassler and D. Schuricht, New J. Phys. 14, 125018 (2012).

[42] L. Fu, Phys. Rev. Lett. 104, 056402 (2010).

[43] K. G. Wilson, Phys. Rev. D 10, 2445 (1974).

[44] J. Kogut and L. Susskind, Phys. Rev. D 11, 395 (1975).

[45] Note that the phase diagram does not depend on the signs of $\lambda, \kappa, E_{M}$.

[46] K. Uzelac, R. Jullien, and P. Pfeuty, Phys. Rev. B 22, 436 (1980).

[47] R. Hützen, A. Zazunov, B. Braunecker, A. L. Yeyati, and R. Egger, Phys. Rev. Lett. 109, 166403 (2012).

[48] In this formula for $K$ we neglect inelastic processes mediated by phonons and assume that the occupation of single-particle states is that of independent particles in thermal equilibrium.

[49] At $\kappa=0$, the ground manifold is $2^{N-1}$-times degenerate, but we continue to choose $|0\rangle$ to be the unique gauge-invariant state in the ground manifold.

[50] C. Lacroix, J. Phys. F: Met. Phys. 11, 2389 (1981).

[51] When the total Hamiltonian is projected onto a single gauge sector, it takes the form of a transverse-field Ising model in a longitudinal field $\kappa$. This projected Hamiltonian is the one we numerically diagonalize. Within this simpler model in a fixed gauge sector, it is well known that any finite $\kappa>0$ introduces a relevant perturbation [46]. 